\title{
Eunomia: Toward a Framework for Multi-touch Information Displays in Public Spaces
}

\author{
Tom Cuypers Jan Schneider Johannes Taelman Kris Luyten Philippe Bekaert \\ Hasselt University - tUL - IBBT \\ Expertise Centre for Digital Media \\ Wetenschapspark 2, 3590 Diepenbeek, Belgium
}

\{tom.cuypers,jan.schneiderbarnes,johannes.taelman,kris.luyten,phillipe.bekaert\}@ uhasselt.be

\begin{abstract}
Multi-touch interaction techniques are becoming more widespread because of new industrial initiatives to make this hardware available and affordable for the consumer market. To cope with the diversity in hardware setups and the lack of knowledge about developing generic multi-touch applications, we created a framework, Eunomia, for abstracting the hardware from the software and to enable software developers to easily develop interactive applications taking advantage of multitouch interaction. We describe our first set of applications created on top of this framework that are targeted for public spaces. During the deployment of these applications, we were able to observe users that are confronted with multi-touch technologies in a public space.
\end{abstract}

\section{Categories and Subject Descriptors}

H.5.3 [Group and Organization Interfaces]: Collaborative computing

\section{General Terms}

Design, Human Factors

\section{Keywords}

Multi-touch, Multi-user interaction, Tabletop

\section{INTRODUCTION}

Nowadays, we are overthrown with huge amounts of information that needs to be accessible in public spaces. In these spaces, there are users with all kinds of backgrounds accessing the information. For example, in most museums there is a lot of digital information available on the exhibit but it is difficult to make them accessible to the audience using traditional devices such as information kiosks with a touchscreen. Interactive computerized systems in public places should take in consideration the special characteristics of these places: everyone can enter them, and there might be many different users at once. The interfaces should be robust enough in order to endure all types of user's interactions and it should support interaction with multiple users simultaneously. Now that multi-touch interfaces have become affordable they seem to be a suitable solution to fill in these requirements.

These multi-touch screens have been tested before in public spaces. For example, for 8 days during summer 2007 in Helsinki a Multi-Touch screen was located in the street using an application where users were able to watch, move and rotate pictures. 1199 persons stop to interact with the screen. This study shows that these types of multi-touch interactions are appealing for a lot of people, and therefore Multi-Touch surfaces are suitable for public spaces [10].

(C) The Author 2008 .

Published by the British Computer Society

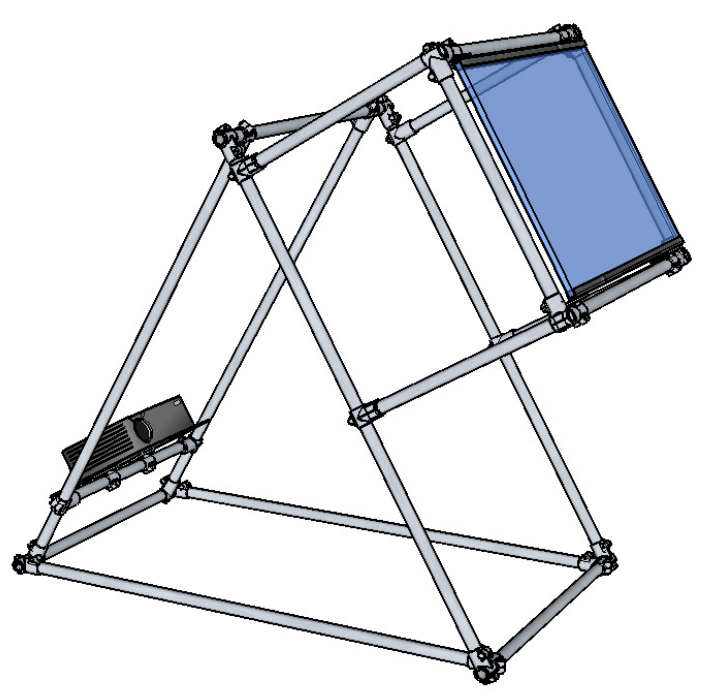

Figure 1: Setup of the multi touch table

We created a multi-touch display that uses the frustrated total internal reflection technique [7] and a software framework to support exploration of the digital information that is related with the public space where the display is installed. Our setup is robust enough for heavy usage in a public space and allows for a vertical display position that makes it easier for users interact with. In combination with this setup we implemented the Eunomia software platform to support the design and development of multi-touch applications. Eunomia provides the basic services for the development of multi-touch applications, such as support for simple physically-based interactions and simultaneous access to multimedia objects. While there are many alternatives available, to our knowledge there are little or no libraries that support a simultaneous multi-user input explicitly. We used Eunomia for the development of a multimedia exploration interface for public spaces.

\section{RELATED WORK}

Although interactive tabletop displays and interactive whiteboards have been around for a long time now, their popularity in public spaces increased during the last couple of years.

Touch screens have been used lately in handy electronic devices such as GPS and cell phones, to name some of them. This implementation has been introduced in order to maximize their display capabilities without the need to increment their size. While these touch screens allow only a single touch at the time, there are some other technologies which allow multi- touch and multi-user interaction. In 1984, Lee et al. implemented an active capacitive sensor matrix capable of tracking multiple touches simultaneously [1]. This early prototype only had a resolution of $64 \times 32$ and suffered from interference between sensors. Some 
other examples of early multi-touch interfaces are the MTC Express [2] and JazzMutant [3]. All of these systems have a complexity on the order of the number of tactels, which limits both resolution and physical scale.

In 2002 Mitsubishi Electric Research Laboratories introduced The Diamond-touch multi-user input surface together with the DiamondSpin Software, allowing a limited amount of identified users to interact together in a digital screen $[4,5,6]$. Each touch produced in the common surface, stores information about its location and the particular user who emitted it.

In 2005, Jeff Han [7] created an optical based multi touch surface. This technology known as the multi-touch sensing through frustrated total internal reflection, allows an unlimited unidentified amount of users to interact on a screen at the same time.

The Microsoft Surface is a well-known example of this technology [8]. It is a 30-inch display in a table-like form, where a small group of people can interact with it by using touch, natural gestures or placing physical objects on the screen.

With the introduction of the Wii Remote by Nintendo in 2006, which contains an infrared camera with built-in hardware blob tracking, some research about Low-Cost Multi-point Interactive Whiteboards is being done, for example by Chung Lee [9].

Multi touch displays are not limited for big screens; its popularity has been growing so fast now even small devices, such as the iPhone count now with this technology.

A study about this new technology performed by Widgor et al. [11] show that replacing mouse and keyboard with a multitouch display in a common office environment is feasible.

Examples of multi-touch interactions that deal with audio information can be found in $[14,15]$ where multi-touch is used to control live audio and [16] that shows a multi-touch music browser. Multi-touch technology creates the opportunity for multi-user interaction and collaboration. Research has been done on how to use 2D gestures as input data for application $[12,13]$.

Even though a multi-touch display is only 2 dimensional, it can often be used to visualize 3D data. On overview of such technologies are described by Grossman et al [17].

Xenakis[18] and reacTIVision[19] propose a framework which is suitable for multiple users, yet their interaction techniques mainly focus on recognizing surface patterns. To our consideration this is less suitable for public spaces, because in this situation it is often better when people interact with their own fingers instead of special objects.

\section{HARDWARE SETUP}

The hardware setup we use is inspired by Jeff Han's implementation [7] and makes use of computer vision techniques to locate the multiple touch points. The screen consists of an acrylic plate in which infrared light is injected. On top of the acrylic there is a compliant surface film and a rear-projection film. Touching the projection film will cause optical contact between the compliant surface and the acrylic, resulting in local scattering of the infrared light. An infrared camera, located below the surface, is able to detect the scattered light.

The compliant surface together with the projection surface reduces the friction otherwise needed for causing FTIR directly with fingers on acrylic. And additionally it removes any parallax effect, since the projection is on the top side of the acrylic.

The compliant surface film softens the surface for more smooth interaction and the projection film solves the parallax issue. It makes sure the perceived distance between your finger and the projected object is minimized. This setup is illustrated in figure 1.

After a brief calibration between the projector and the camera, the coordinates and size of all the touch points can be obtained correctly. Our setup detects and arbitrary amount of touch points and communicates them with interested client systems using UDP messages. As this is the connection with the software running on the multi-touch, we will elaborate further on this in the next section

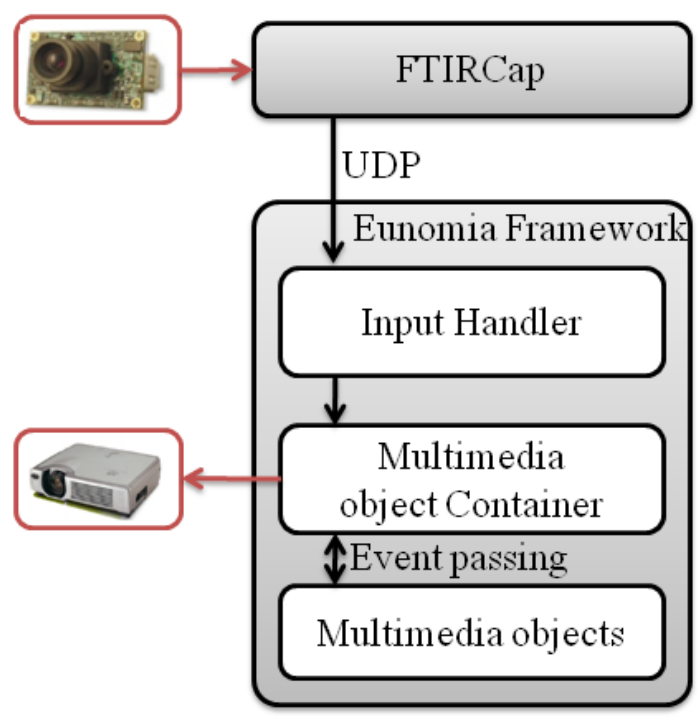

Figure 2: Software Architecture Overview

\section{SOFTWARE ARCHITECTURE}

Our software framework, Eunomia, was originally developed for displaying and manipulating multimedia data on a multitouch interface. The framework is subdivided in two independent parts: the input-handler takes care of the touch events and the multimedia object container enables application developers to create interactive applications using multimedia objects. The former essentially captures the inputs from the user and transforms them into events. The latter deals with visualization and manipulation of multimedia objects using multiple touch points. A brief overview of how the framework is used can be found in figure 2. One of the most important goals of the framework is to abstract the hardware being used, so other types of multi-touch technologies can be easily integrated.

As previously explained, the touch points are detected using an infrared camera. The captured frames are analyzed and converted into touch-points in a separate executable called FTIRCap. Each control-point consists of its screen coordinates, its size and intensity (the pressure that is put on the table). The camera/projection calibration is also handled in this executable. These touch-points are then streamed over UDP, making it possible to run this module on a different computer. The whole image capture, analysis and transmission occur at a frequency of $60 \mathrm{~Hz}$, for fluent low-latency interaction. Because of the lowcost processing of the captured images, our applications can easily run on a single desktop computer.

The input-handler first receives the touch-points and converts them into control-points. The difference between a touch-point and a control-point is that the control -point has a unique identifier persistent over one continuous stroke on the screen. The received set of touch-points is compared to the set of control points to update their attributes. 
The multimedia object container is the main module. It handles events from, to and between multimedia-objects. For example, it associates the control-points to the objects and it handles the rendering for which it uses OpenGL.

Specific multi-touch applications are implemented by using the multimedia object container and instancing a set of multimedia objects. These objects use functionality provided by Eunomia for displaying and event handling.

Some example multimedia objects are dedicated to videos, images, buttons and collection browsers.

This architecture allows the multimedia objects to be completely hardware independent; each one of them is able to receive input from different input devices simultaneously. This framework is also general enough for describing different interaction techniques. Using these events, different kinds of interactions can be defined for the different multimedia objects. Due to its scalability, the Eunomia platform has been successfully used to develop new applications. Users not familiar with this framework were able to create new multimedia objects and applications on it, which afterwards were tested in small demonstrations.

\section{MULTITOUCH APPLICATIONS}

In this section we discuss three applications on the multi-touch table, a photo-browser, a video player and a digital book library

\subsection{Photo-browser}

A first example application made with our framework is a photo-browser where the multimedia objects are images. The implementation of the control-point events allows the user to transform the images with one or two fingers. The user can translate, rotate and scale the images by moving their fingers on them, where the relative positions of the associated contact points towards the image are preserved as illustrated in figure 3. This interaction technique is intuitive since it follows a direct manipulation, naïve physics metaphor.

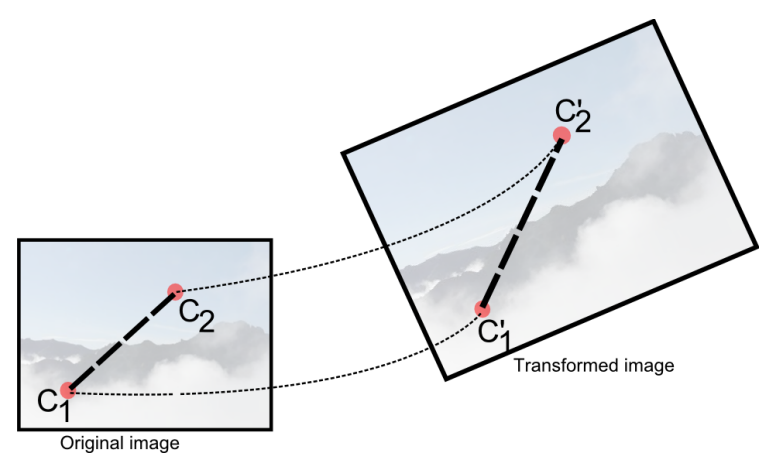

Figure 3: Transforming images in the photo-browser

\subsection{Video Manipulation and Selection}

An extension to the photo-browser is allowing users to view and navigate through videos. This multimedia-object can be displayed as constantly changing images.

A common way to navigate through a video is using a scrollbar or a timeline. Looking for a specific event in a sequence is not always that simple if you don't know when this happened. In this application we propose the metaphor of a flipbook. When holding the video, sample video frames can be extracted. They are laid out on a fan-like shape as shown on figure 4. Sample frames before the current play time of the video are shown on the left and frames after this time on the right. Clicking on a specific frame will navigate you to its corresponding time in the video.

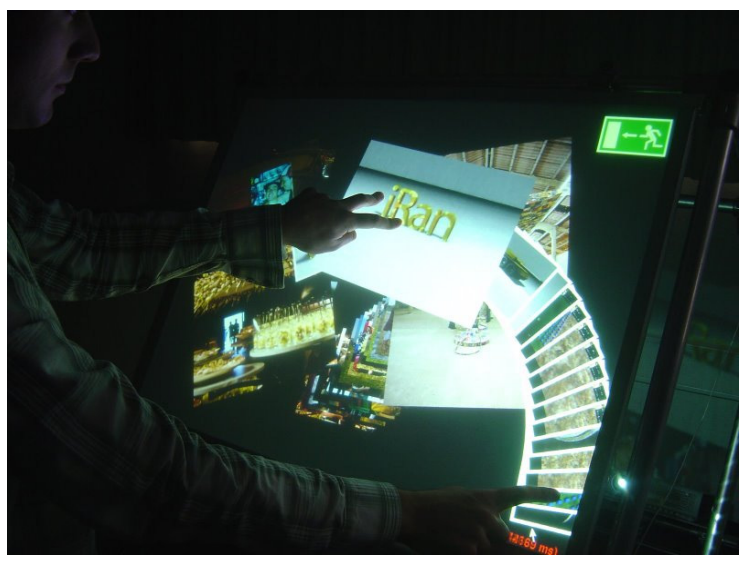

Figure 4: Video browsing

The first component is a book browser. It contains an on-screen keyboard by which the user can search for a specific book from Google Books ${ }^{1}$, and an area where the search results coming from Google Books are shown.

The second part of the library application is the representation of the book itself. A book is a set of images and each image represents a page of the book. The book is displayed on the multi-touch table as a traditional open book, where two continuous pages are always shown.

The book can be resized and rotated as any other multimediaobject by using two touch points .In order to browse through its pages three touch points are required to differentiate from resizing/rotating. Two fingers to hold the book, and a third finger moving across the fold will scroll to the previous or next page depending on the direction of that movement as shown on figure 5 .

\section{DISCUSSION}

During our research we found that, the hardware used for our multi-touch screen proved to be robust enough to be used in public spaces. We successfully demonstrated the applications described in this paper in the library of Ghent University (Belgium) and the Arts Center Vooruit also in Ghent.

We observed usage of the multi-touch display while it was deployed at the arts center. Although we did not do any usability test, users seems to find using the multi-touch display both appealing and fun. One example is our implementation

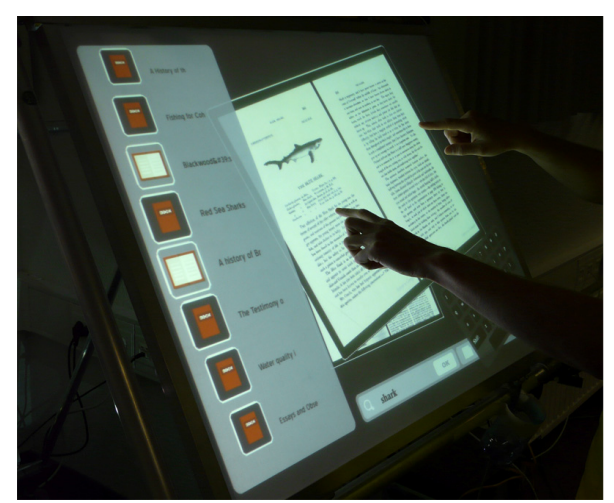

Figure 5: Library application

\footnotetext{
${ }^{1} \mathrm{http} / / /$ books.google.com/
} 
that provided multi-touch interaction with Google Earth ${ }^{2}$. Nearly all users tried to find their house by navigating through the map using both hands (and multiple fingers). These users had different backgrounds, ranging from people that have experience using Google Earth to others did never hear from this application. We find that most users tend to use both hands and multiple fingers intuitively when interacting with the applications described in this paper.

Multi-touch screens not only present a new set of interactions that are appealing for the people, multi-touch screens have also the capability to allow multiple persons to interact at the same time with it, making them a social technology. Our deployments were often used by multiple people at once but not simultaneously touching it. This causes animated discussions on what was presented on screen. It is only after a short period of usage users tends to find out it can be used by multiple users simultaneously. A remark often heard during usage by multiple people is "Can I try now?", while waiting for the active user retracting her hands of the screen. This indicates the user interface should make users aware that it can support simultaneous interaction.

\section{ACKNOWLEDGMENTS}

Part of the research at EDM is funded by EFRO (European Fund for Regional Development) and the Flemish Government. We also like to thank Henk Catry of the Vooruit in Ghent for his time and effort in this work.

\section{REFERENCES}

[1] SK. Lee, W. Buxton, K.C. Smith. A Multi-Touch Three dimensional Touch-Sensitive Tablet. Proceedings of the SIGCHI conference on Human factors in computing systems. 1985.

[2] Tactex. Smart fabric Technology. http://www.tactex.com

[3] JazzMutant. Lemur. http://www.jazzmutant.com. 2004

[4] Scott, S. D. and Carpendale, S. Interacting with Digital Tabletops. IEEE Computer Graphics and Applications. 2006 issue 5.

[5] P. Dietz and D. Leigh. DiamondTouch: A Multi-User Touch Technology. ACM Symposium on User Interface Software and Technology (UIST). November 2001

[6] A. Esenther, C. Forlines, K. Ryall and S. Shipman. DiamondTouch SDK: Support for Multi-User, MultiTouch Applications, Demonstration, CSCW, November 2002.

[7] Han, J. Y. 2005. Low-Cost Multi-Touch Sensing through Frustrated Total Internal Reflection. In Proceedings of the 18th Annual ACM Symposium on User Interface Software and Technology

[8] Microsoft Corp. "The History of Microsoft Surface The making of Microsoft's first commercially available surface computer." May 2007.

[9] Chung Lee, J. "Low-Cost Multi-point Interactive Whiteboards Using the Wiimote", http://www.cs.cmu.edu/ johnny/projects/wii/. $\quad$ February 2008.

[10] Peter Peltonen, Esko Kurvinen, Antti Salovaara, Giulio Jacucci, Tommi Ilmonen: "It's Mine, Don't Touch!": Interactions at a Large Multi-Touch Display in a City Centre. CHI 2008 Proceedings · Multitouch and Surface Computing, April 2008.
[11] D. Wigdor, G. Penn, K. Ryall, A. Esenther and C. Shen. Living with a Tabletop: Analysis and Observations of Long Term Office Use of a Multi-Touch Table. Second Annual IEEE International Workshop on Volume, October 2007

[12] W. Westerman and J.G. Elias. Multi-Touch: A New Tactile 2-D Gesture Interface for Human-Computer Interaction. Human Factors and Ergonomics Society $45^{\text {th }}$ Annual Meeting. 2001.

[13] Vladimir I. Pavlovic, Rajeev Sharma, Thomas S. Huang. Visual Interpretation of Hand Gestures for HumanComputer Interaction: A Review. IEEE Transactions on Pattern Analysis and Machine Intelligence, July 1997

[14] P. L. Davidson and J. Y. Han. Synthesis and Control on Large Scale Multi-Touch Sensing Displays. International Conference on New Interfaces for Musical Expression. France, 2006

[15] J. Patten, B. Recht and H. Ishii. Interaction Techniques for Musical Performance with Tabletop Tangible Interfaces. SIGCHI international conference on Advances in computer entertainment technology. 2006

[16] S. Hitchner, J. Murdoch and G. Tzanetakis. Music Browsing Using a Tabletop Display. 8th International Conference on Music Information Retrieval. September 2007

[17] T. Grossman and D. Wigdor. Going Deeper: a Taxonomy of 3D on the Tabletop. Second Annual IEEE International Workshop on Horizontal Interactive Human-Computer System, 2007

[18] M. Bishof, B. Conradi: Xenakis - Combining tangible interaction with probability - based musical composition. Proceedings of the Second international Conference on Tangible Embedded Interaction, February 2008.

[19] M. Kaltenbrunner, R. Bencina: reacTIVision: A ComputerVision Framework for Table-Based Tangible Interaction. Proceedings of the $1^{\text {st }}$ international conference on Tangible and embedded interaction, February 2007.

${ }^{2}$ http://earth.google.com/ 